\title{
Statistics for Ratios of Rayleigh, Rician, Nakagami- $m$, and Weibull Distributed Random Variables
}

\author{
Dragana Č. Pavlović, ${ }^{1}$ Nikola M. Sekulović, ${ }^{2}$ Gradimir V. Milovanović, ${ }^{3}$ \\ Aleksandra S. Panajotović, ${ }^{4}$ Mihajlo Č. Stefanović, ${ }^{4}$ and Zoran J. Popović ${ }^{5}$ \\ ${ }^{1}$ Faculty of Philosophy, University of Niš, Ćirila i Metodija 2, $18000 \mathrm{Niš}$, Serbia \\ ${ }^{2}$ The School of Higher Technical Professional Education, A. Medvedeva 20, 18000 Niš, Serbia \\ ${ }^{3}$ Mathematical Institute, Serbian Academy of Science and Arts, Kneza Mihaila 36, 11001 Belgrade, Serbia \\ ${ }^{4}$ Faculty of Electronic Engineering, University of Niš, A. Medvedeva 14, 18000 Niš, Serbia \\ ${ }^{5}$ The School of Higher Technical Professional Education, Nušićeva 6, 38227 Zvečan, Serbia \\ Correspondence should be addressed to Gradimir V. Milovanović; gvm@mi.sanu.ac.rs
}

Received 29 June 2013; Revised 29 October 2013; Accepted 5 November 2013

Academic Editor: Yuri Vladimirovich Mikhlin

Copyright (c) 2013 Dragana Č. Pavlović et al. This is an open access article distributed under the Creative Commons Attribution License, which permits unrestricted use, distribution, and reproduction in any medium, provided the original work is properly cited.

\begin{abstract}
The distributions of ratios of random variables are of interest in many areas of the sciences. In this brief paper, we present the joint probability density function (PDF) and PDF of maximum of ratios $\mu_{1}=R_{1} / r_{1}$ and $\mu_{2}=R_{2} / r_{2}$ for the cases where $R_{1}, R_{2}, r_{1}$, and $r_{2}$ are Rayleigh, Rician, Nakagami- $m$, and Weibull distributed random variables. Random variables $R_{1}$ and $R_{2}$, as well as random variables $r_{1}$ and $r_{2}$, are correlated. Ascertaining on the suitability of the Weibull distribution to describe fading in both indoor and outdoor environments, special attention is dedicated to the case of Weibull random variables. For this case, analytical expressions for the joint PDF, PDF of maximum, PDF of minimum, and product moments of arbitrary number of ratios $\mu_{i}=R_{i} / r_{i}, i=1, \ldots, L$ are obtained. Random variables in numerator, $R_{i}$, as well as random variables in denominator, $r_{i}$, are exponentially correlated. To the best of the authors' knowledge, analytical expressions for the PDF of minimum and product moments of $\left\{\mu_{i}\right\}_{i=1}^{L}$ are novel in the open technical literature. The proposed mathematical analysis is complemented by various numerical results. An application of presented theoretical results is illustrated with respect to performance assessment of wireless systems.
\end{abstract}

\section{Introduction}

Radio-wave propagation through wireless channels is a complicated phenomenon characterized by fading which is the result of multipath propagation. When a received signal envelope experiences fading during transmission, it fluctuates over time. Multivariate statistics is a useful mathematical tool for modeling and analyzing wireless channels. There is a very wide range of statistical models for fading channels [1] which their accuracy and veracity depend on propagation environment and communication scenario. Rayleigh, Rician, Nakagami- $m$, and Weibull are the most frequently applied models in the open technical literature.

Fading can seriously degrade performance of wireless communications systems. Techniques that can be used to minimize the degradation effects due to fading have received a great deal of research interest. Diversity combining [1], which combines two or more replicas of the received signal, is a practical and powerful technique that can be used to alleviate the detrimental effects of fading and to improve the performance of wireless communications systems without increasing transmission power and bandwidth. Space diversity [2], achieved by using multiple antennas at the receiver, is the most common form of diversity. The most popular space diversity techniques are selection combining (SC), equal-gain combining (EGC), and maximal-ratio combining (MRC).

In digital contemporary communications systems, fading channels are correlated due to insufficient antenna spacing when diversity is applied in small terminals (e.g., hand-held mobile terminals and compact base stations). Several spatial correlation models have been proposed and used for the performance analysis of various wireless systems, corresponding 
to specific modulation, detection, and diversity schemes [1]. Spatial exponential correlation model is one of the most frequently used in performance analysis of wireless systems with multichannel reception [3-5]. The correlation matrix of this model is described by $\sum_{i j} \equiv \rho^{|i-j|}, i, j=1, \ldots, L$, where $\rho(0 \leq$ $\rho<1)$ is the correlation coefficient between adjacent channels [1, Equation (9.164)] and $L$ is the number of channels. The exponential correlation model corresponds to the scenario of multichannel reception from equispaced diversity antennas in which the correlation among the pairs of combined signals decays as the spacing between the antennas increases.

The distribution of the ratio of random variables is of interest in statistical analysis in biological and physical sciences, econometrics, and ranking and selection [6]. It has been studied by several authors especially when random variables are independent and come from the same family [7-10]. In [11, 12], the distribution of the ratio of correlated random variables is considered.

The ratios of random variables are also of interest in analyzing wireless communications systems in fading environment [13-17]. Namely, the random variable in nominator may present desired signal envelope while the random variable in denominator may present interference signal envelope. Rayleigh, Rician, Nakagami- $m$, and Weibull distributions are included in our analysis. Having in mind that it is well known that the assumption of independence among the input diversity channels is not accurate for compact, hand-held, mobile terminals and indoor base stations with no sufficiently separated antennas, random variables in nominator, as well as random variables in denominator, are correlated. It shows that results presented in the paper can be efficiently used in analyzing realistic correlated fading channels.

\section{On Two Ratios of Rayleigh, Rician, and Nakagami- $m$ Random Variables}

In this section, the joint probability density function (PDF) and PDF of maximum of two ratios of random variables $\mu_{1}=$ $R_{1} / r_{1}$ and $\mu_{2}=R_{2} / r_{2}$ are presented. Random variables $R_{1}$ and $R_{2}$, as well as random variables $r_{1}$ and $r_{2}$, are correlated. The joint PDF of $\mu_{1}$ and $\mu_{2}$ can be obtained as

$$
\begin{aligned}
& p_{\mu_{1} \mu_{2}}\left(\mu_{1}, \mu_{2}\right) \\
& \quad=\iint_{0}^{\infty}|J| p_{R_{1} R_{2}}\left(\mu_{1} r_{1}, \mu_{2} r_{2}\right) p_{r_{1} r_{2}}\left(r_{1}, r_{2}\right) d r_{1} d r_{2},
\end{aligned}
$$

where $p_{R_{1} R_{2}}\left(R_{1}, R_{2}\right)$ and $p_{r_{1} r_{2}}\left(r_{1}, r_{2}\right)$ are the joint PDFs of random variables in nominator and denominator, respectively, and $|J|$ is the Jacobian transformation given by

$$
|J|=\left|\begin{array}{ll}
\frac{d R_{1}}{d \mu_{1}} & \frac{d R_{1}}{d \mu_{2}} \\
\frac{d R_{2}}{d \mu_{1}} & \frac{d R_{2}}{d \mu_{2}}
\end{array}\right|=r_{1} r_{2}
$$

The PDF expression of maximum of ratios of random variables $\mu_{\max }=\max \left\{\mu_{1}, \mu_{2}\right\}$ can be derived as

$$
p_{\mu_{\max }}(\mu)=\int_{0}^{\mu} p_{\mu_{1} \mu_{2}}\left(\mu, \mu_{2}\right) d \mu_{2}+\int_{0}^{\mu} p_{\mu_{1} \mu_{2}}\left(\mu_{1}, \mu\right) d \mu_{1} \text {. }
$$

In the analysis of wireless communications systems, depending on the nature of the radio propagation environment, there are different models describing the statistical behavior of the multipath fading envelope. In the rest of this section, correlative Rayleigh, Rician, and Nakagami- $m$ fading models are considered. The existence of correlation is the real scenario in practical multiantennas wireless systems due to insufficient antenna spacing. Since, this paper considers spatial correlation-the correlation between appropriate pair of receive antennas-all signals, regardless of their nature, received by these antennas explore the same correlation coefficient.

(a) Rayleigh Case. The Rayleigh distribution is frequently used to model multipath fading with no direct line-of-sight (LOS) path. The joint bivariate PDFs of correlated Rayleigh distributed random variables $R_{i}$ and $r_{i}(i=1,2)$ are given by $[1$, Equation (6.2)] as follows:

$$
\begin{aligned}
p_{R_{1} R_{2}}\left(R_{1}, R_{2}\right)= & \frac{4 R_{1} R_{2}}{\Omega_{d 1} \Omega_{d 2}(1-\rho)} \\
& \times \exp \left[-\frac{1}{1-\rho}\left(\frac{R_{1}^{2}}{\Omega_{d 1}}+\frac{R_{2}^{2}}{\Omega_{d 2}}\right)\right] \\
& \times I_{0}\left[\frac{2 \sqrt{\rho} R_{1} R_{2}}{(1-\rho) \sqrt{\Omega_{d 1} \Omega_{d 2}}}\right], \\
p_{r_{1} r_{2}}\left(r_{1}, r_{2}\right)= & \frac{4 r_{1} r_{2}}{\Omega_{c 1} \Omega_{c 2}(1-\rho)} \\
& \times \exp \left[-\frac{1}{1-\rho}\left(\frac{r_{1}^{2}}{\Omega_{c 1}}+\frac{r_{2}^{2}}{\Omega_{c 2}}\right)\right] \\
& \times I_{0}\left[\frac{2 \sqrt{\rho} r_{1} r_{2}}{(1-\rho) \sqrt{\Omega_{c 1} \Omega_{c 2}}}\right],
\end{aligned}
$$

where $I_{0}(\cdot)$ is the modified Bessel function of the first kind and zero order, $\rho$ is the correlation coefficient, and $\Omega_{d i}$ and $\Omega_{c i}$ are the mean-square values of $R_{i}$ and $r_{i}$, respectively. Substituting (2) and (4) in (1) and using the infinite-series representation of the modified Bessel function [18, Equation $(8.447 / 1)]$, integrals can be solved with the aid of [18, Equation (3.478/1)]. Analytical expression for the joint PDF of $\mu_{1}$ and $\mu_{2}$ can be finally written as

$$
\begin{aligned}
& p_{\mu_{1} \mu_{2}}\left(\mu_{1}, \mu_{2}\right) \\
& =4(1-\rho)^{2} \sum_{k, \ell=0}^{\infty} \frac{\rho^{k+\ell}\left(\mu_{1} \mu_{2}\right)^{2 k+1} \Gamma^{2}(2+k+\ell)}{\left(\Omega_{d 1} \Omega_{d 2}\right)^{1+k}\left(\Omega_{c 1} \Omega_{c 2}\right)^{1+\ell}(k ! \ell !)^{2}} \\
& \times\left(\left(\frac{\mu_{1}^{2}}{\Omega_{d 1}}+\frac{1}{\Omega_{c 1}}\right)\left(\frac{\mu_{2}^{2}}{\Omega_{d 2}}+\frac{1}{\Omega_{c 2}}\right)\right)^{-(2+k+\ell)} .
\end{aligned}
$$


Substituting (5) in (3) and using [18, Equation (3.194/1)], the PDF of maximum of two ratios of Rayleigh random variables can be written as

$$
\begin{aligned}
& p_{\mu_{\max }}(\mu) \\
&=(1-\rho)^{2} \sum_{k, \ell=0}^{\infty} \frac{\rho^{k+\ell} \Gamma^{2}(2+k+\ell) \mu^{4 k+3}}{(k ! \ell !)^{2}(1+k)}\left(\frac{\Omega_{c 1} \Omega_{c 2}}{\Omega_{d 1} \Omega_{d 2}}\right)^{1+k} \\
& \times\left\{\frac{2}{\left(1+\left(\left(\Omega_{c 1} / \Omega_{d 1}\right) \mu^{2}\right)\right)^{2+k+\ell}}\right. \\
& \quad \times{ }_{2} F_{1}\left(2+k+\ell, 1+k ; 2+k ;-\frac{\Omega_{c 2}}{\Omega_{d 2}} \mu^{2}\right) \\
&+\frac{2}{\left(1+\left(\left(\Omega_{c 2} / \Omega_{d 2}\right) \mu^{2}\right)\right)^{2+k+\ell}} \quad \\
&\left.\quad \times{ }_{2} F_{1}\left(2+k+\ell, 1+k ; 2+k ;-\frac{\Omega_{c 1}}{\Omega_{d 1}} \mu^{2}\right)\right\}
\end{aligned}
$$

where ${ }_{2} F_{1}(a, b ; c ; d)$ is the Gauss hypergeometric function.

(b) Rician Case. The Rician distribution is often used to model propagation paths consisting of one strong direct LOS component and many random weaker components. In this case, $R_{i}$ and $r_{i}(i=1,2)$ are distributed according to [15, Equation (1)] as follows:

$$
\begin{aligned}
p_{R_{1} R_{2}}\left(R_{1}, R_{2}\right) & \frac{4 R_{1} R_{2}(K+1)^{2}}{\Omega_{d}^{2}\left(1-\rho^{2}\right)} \\
& \times \exp \left(-\frac{\left(R_{1}^{2}+R_{2}^{2}\right)(K+1)+2 K \Omega_{d}(1-\rho)}{\Omega_{d}\left(1-\rho^{2}\right)}\right) \\
& \times \sum_{k=0}^{\infty} \varepsilon_{k} I_{k}\left(\frac{2 R_{1} R_{2} \rho(K+1)}{\Omega_{d}\left(1-\rho^{2}\right)}\right) \\
& \times I_{k}\left(\frac{2 R_{1}}{1+\rho} \sqrt{\frac{K(K+1)}{\Omega_{d}}}\right) I_{k}\left(\frac{2 R_{2}}{1+\rho} \sqrt{\frac{K(K+1)}{\Omega_{d}}}\right), \\
= & \left.\frac{4 r_{1} r_{2}(K+1)^{2}}{\Omega_{c}^{2}\left(1-\rho^{2}\right)}, r_{2}\right) \\
& \times \exp \left(-\frac{\left(r_{1}^{2}+r_{2}^{2}\right)(K+1)+2 K \Omega_{c}(1-\rho)}{\Omega_{c}\left(1-\rho^{2}\right)}\right) \\
& \times \sum_{\ell=0}^{\infty} \varepsilon_{\ell} I_{\ell}\left(\frac{2 r_{1} r_{2} \rho(K+1)}{\Omega_{c}\left(1-\rho^{2}\right)}\right) \\
& \times I_{\ell}\left(\frac{2 r_{1}}{1+\rho} \sqrt{\frac{K(K+1)}{\Omega_{c}}}\right)
\end{aligned}
$$

where $I_{n}(\cdot)$ is the modified Bessel function of the first kind and $n$th order, $\varepsilon_{0}=1, \varepsilon_{k}=2, k \geq 1, \Omega_{d}=\overline{R_{i}^{2}}, \Omega_{c}=\overline{r_{i}^{2}}$, and $K$ is the Rician factor. The Rician distribution spans the range from Rayleigh fading $(K \rightarrow 0)$ to no fading $(K \rightarrow \infty)$. Following the same procedure as for the Rayleigh case, the joint PDF of $\mu_{1}$ and $\mu_{2}$ becomes [17, Equation (6)] as follows:

$$
\begin{gathered}
p_{\mu_{1} \mu_{2}}\left(\mu_{1}, \mu_{2}\right) \\
=\frac{\exp (-4 K /(1+\rho))}{(K+1)^{2}} \\
\times \sum_{k, \ell, m, n, p, q, s, w=0}^{\infty} \frac{\mu_{1}^{2(m+n+k)} \Omega_{d}^{2(q+\ell+1)+s+w} \Omega_{c}^{2(m+k+1)+n+p}}{(1+\rho)^{2(n+p+k+s+w+\ell)} m ! n ! p ! q ! s ! w !} \\
\times\left(\rho^{2(m+q)+k+\ell} \mu_{2}^{2(m+p+k)}\left(1-\rho^{2}\right)^{n+p+k+s+w+\ell+2}\right. \\
\quad \times \varepsilon_{k} \varepsilon_{\ell} K^{n+p+k+s+w+\ell} E(k, \ell, m, n, p, q, s, w) \\
\times\left(\left(\Omega_{d}+\Omega_{c} \mu_{1}^{2}\right)^{m+n+k+q+s+\ell+2}\right. \\
\left.\left.\times\left(\Omega_{d}+\Omega_{c} \mu_{2}^{2}\right)^{m+p+k+q+w+\ell+2}\right)^{-1}\right),
\end{gathered}
$$

where $E(k, \ell, m, n, p, q, s, w) \equiv E$ is given by

$$
\begin{aligned}
E= & \Gamma(m+n+k+q+s+\ell+2) \\
& \times \Gamma(m+p+k+q+w+\ell+2) \\
& \times(\Gamma(m+k+1) \Gamma(n+k+1) \Gamma(p+k+1) \\
& \quad \times \Gamma(q+\ell+1) \Gamma(s+\ell+1) \Gamma(w+\ell+1))^{-1},
\end{aligned}
$$

while the PDF of $\mu_{\max }$ can be written as in [17, Eq. (11)] as

$$
\begin{aligned}
& p_{\mu_{\max }}(\mu) \\
& =\exp \left(-\frac{4 K}{1+\rho}\right) \\
& \times \sum_{k, \ell, m, n, p, q, s, w=0}^{\infty}\left(\varepsilon_{k} \varepsilon_{l} K^{n+p+k+s+w+\ell} \rho^{2(m+q)+k+\ell}\right. \\
& \times\left(1-\rho^{2}\right)^{n+p+k+s+w+\ell+2} \\
& \left.\times\left((1+\rho)^{2(n+p+k+s+w+\ell)} m ! n ! p ! q ! s ! w !\right)^{-1}\right) \\
& \times \frac{\mu^{4(m+k)+2(p+n+1)} S^{q+\ell+1}}{\left(S+\mu^{2}\right)^{2(k+m)+n+p+\ell+q+3}} E(k, \ell, m, n, p, q, s, w) \\
& \times\left[( \frac { S } { S + \mu ^ { 2 } } ) ^ { w } \left({ }_{2} F_{1}(m+n+k+1,-\ell-q-s ;\right.\right. \\
& \left.m+n+k+2 ; \frac{\mu^{2}}{\mu^{2}+S}\right) \\
& \left.\times(m+n+k+1)^{-1}\right)
\end{aligned}
$$




$$
\begin{gathered}
+\left(\frac{S}{S+\mu^{2}}\right)^{s}\left({ }_{2} F_{1}(m+p+k+1,-\ell-q-w ;\right. \\
\left.m+p+k+2 ; \frac{\mu^{2}}{\mu^{2}+S}\right) \\
\left.\left.\times(m+p+k+1)^{-1}\right)\right]
\end{gathered}
$$

where $S=\Omega_{d} / \Omega_{c}$ and $E$ is defined before.

(c) Nakagami-m Case. The Nakagami-m distribution has gained widespread application in the modeling of physical fading radio channels [19]. The primary justification of the use of Nakagami- $m$ fading model is its good fit to empirical fading data. It is versatile and through its parameter $m$, we can model signal fading conditions that range from severe to moderate, to light fading, or no fading. It includes the one-sided Gaussian distribution $(m=0.5)$ and the Rayleigh distribution $(m=1)$ as special cases. In Nakagami- $m$ fading environment, $R_{i}$ and $r_{i}(i=1,2)$ follow the distributions

$$
\begin{aligned}
p_{R_{1} R_{2}}\left(R_{1}, R_{2}\right)= & \frac{4 m_{1}^{m_{1}+1}\left(R_{1} R_{2}\right)^{m_{1}}}{\Gamma\left(m_{1}\right) \Omega_{d 1} \Omega_{d 2}(1-\rho)\left(\sqrt{\rho \Omega_{d 1} \Omega_{d 2}}\right)^{m_{1}-1}} \\
& \times \exp \left(-\frac{m_{1}\left(R_{1}^{2}+R_{2}^{2}\right)}{\sqrt{\Omega_{d 1} \Omega_{d 2}}(1-\rho)}\right) \\
& \times I_{m_{1}-1}\left(\frac{2 \sqrt{\rho} m_{1} R_{1} R_{2}}{\sqrt{\Omega_{d 1} \Omega_{d 2}}(1-\rho)}\right), \\
p_{r_{1} r_{2}}\left(r_{1}, r_{2}\right)= & \left.\frac{4 m_{2}^{m_{2}+1}\left(r_{1} r_{2}\right)^{m_{2}}}{\Gamma\left(m_{2}\right) \Omega_{c 1} \Omega_{c 2}(1-\rho)\left(\sqrt{\rho} \Omega_{c 1} \Omega_{c 2}\right.}\right)^{m_{2}-1} \\
& \times \exp \left(-\frac{m_{2}\left(r_{1}^{2}+r_{2}^{2}\right)}{\sqrt{\Omega_{c 1} \Omega_{c 2}}(1-\rho)}\right) \\
& \times I_{m_{2}-1}\left(\frac{2 \sqrt{\rho} m_{2} r_{1} r_{2}}{\sqrt{\Omega_{c 1} \Omega_{c 2}}(1-\rho)}\right) .
\end{aligned}
$$

Setting

$$
\begin{aligned}
W= & \left(\frac{m_{1} \mu_{1}^{2}}{\sqrt{\Omega_{d 1} \Omega_{d 2}}}+\frac{m_{2}}{\sqrt{\Omega_{c 1} \Omega_{c 2}}}\right) \\
& \times\left(\frac{m_{1} \mu_{2}^{2}}{\sqrt{\Omega_{d 1} \Omega_{d 2}}}+\frac{m_{2}}{\sqrt{\Omega_{c 1} \Omega_{c 2}}}\right),
\end{aligned}
$$

the joint PDF of ratios of the Nakagami- $m$ distributed random variables can be obtained as

$$
\begin{gathered}
p_{\mu_{1} \mu_{2}}\left(\mu_{1}, \mu_{2}\right) \\
=\frac{4(1-\rho)^{m_{1}+m_{2}}}{\Gamma\left(m_{1}\right) \Gamma\left(m_{2}\right)} \\
\quad \times \sum_{k, l=0}^{\infty}\left(\rho^{k+l} \Gamma^{2}\left(k+l+m_{1}+m_{2}\right)\right. \\
\quad \times\left(\mu_{1} \mu_{2}\right)^{2\left(k+m_{1}\right)-1} m_{1}^{2\left(m_{1}+k\right)} m_{1}^{2\left(m_{2}+l\right)} \\
\quad \times\left(k ! l ! \Gamma\left(k+m_{1}\right) \Gamma\left(l+m_{2}\right)\left(\Omega_{c 1} \Omega_{c 2}\right)^{l+m_{2}}\right. \\
\left.\left.\quad \times\left(\Omega_{d 1} \Omega_{d 2}\right)^{k+m_{1}} W^{k+l+m_{1}+m_{2}}\right)^{-1}\right),
\end{gathered}
$$

while the PDF of maximum of ratios of Nakagami- $m$ distributed random variables is

$$
\begin{aligned}
& p_{\mu_{\max }}(\mu) \\
& =\frac{4(1-\rho)^{m_{1}+m_{2}}}{\Gamma\left(m_{1}\right) \Gamma\left(m_{2}\right)} \\
& \times \sum_{k, l=0}^{\infty}\left(\rho^{k+l} \Gamma^{2}\left(k+l+m_{1}+m_{2}\right)\right. \\
& \times \mu^{4\left(k+m_{1}\right)-1}\left(\Omega_{c 1} \Omega_{c 2}\right)^{\left(m_{1}+k-m_{2}-l\right) / 2} \\
& \times m_{1}^{2\left(m_{1}+k\right)} m_{2}^{\left(m_{2}+l-m_{1}-k\right)} \\
& \times\left(k ! l ! \Gamma\left(k+m_{1}\right) \Gamma\left(l+m_{2}\right)\right. \\
& \times\left(k+m_{1}\right)\left(\Omega_{d 1} \Omega_{d 2}\right)^{\left(m_{1}+k\right)} \\
& \left.\left.\times\left(\frac{\mu^{2} m_{1}}{\sqrt{\Omega_{d 1} \Omega_{d 2}}}+\frac{m_{2}}{\sqrt{\Omega_{c 1} \Omega_{c 2}}}\right)^{k+l+m_{1}+m_{2}}\right)^{-1}\right) \\
& \times{ }_{2} F_{1}\left(k+l+m_{1}+m_{2}, m_{1}+k ; k+m_{1}+1 ;\right. \\
& \left.\frac{-\mu^{2} m_{1} \sqrt{\Omega_{c 1} \Omega_{c 2} / \Omega_{d 1} \Omega_{d 2}}}{m_{2}}\right) .
\end{aligned}
$$


The above presented results can be efficiently used for analyzing wireless communications systems in fading environment. Namely, the joint bivariate PDF of ratios of random variables is necessary for performance analysis of dual diversity combining schemes. In that case, $\mu_{i}$ denotes signalto-interference ratio, $\Omega_{d i}$ and $\Omega_{c i}$ are the average powers of desired and interference signals at $i$ th diversity branch, respectively, and $\rho$ is the correlation between branches. Varying the Rician and Nakagami- $m$ parameter, it is possible to simulate fading with different severity degrees. Having in mind that in interference-limited environment SC diversity receiver selects and outputs the branch with the highest signal-to-interference ratio, the PDF of $\mu_{\max }$ presents the PDF of signal-to-interference ratio at the SC output. This expression can be used to study important performance measures such as the average bit error probability and channel capacity.

\section{On the Ratios of Weibull Distributed Random Variables}

The Weibull distribution exhibits an excellent fit to experimental fading channel measurements, for both indoor [20] and outdoor [21, 22] environments. It is the reason why Weibull distribution paved its way to wireless communications applications. The fact that the diversity receiver with larger number of branches shows better performance gives an idea to investigate the statistics of arbitrary number of ratios. In this section, the joint PDF, product moments, and PDF of maximum and minimum of arbitrary number of ratios of Weibull distributed random variables are presented. To the best of the authors' knowledge, analytical expressions for product moments and PDF of minimum of ratios of Weibull random variables are novel in the open technical literature.

(a) The Joint PDF. The joint bivariate PDFs of correlated Weibull distributed random variables $R_{i}$ and $r_{i}(i=1,2)$ are given by [23, Equation (11)] as follows:

$$
\begin{aligned}
p_{R_{1} R_{2}}\left(R_{1}, R_{2}\right)= & \frac{\beta_{1} \beta_{2} R_{1}^{\beta_{1}-1} R_{2}^{\beta_{2}-1}}{\Omega_{d 1} \Omega_{d 2}(1-\rho)} \\
& \times \exp \left[-\frac{1}{1-\rho}\left(\frac{R_{1}^{\beta_{1}}}{\Omega_{d 1}}+\frac{R_{2}^{\beta_{2}}}{\Omega_{d 2}}\right)\right] \\
& \times I_{0}\left[\frac{2 \sqrt{\rho} R_{1}^{\beta_{1} / 2} R_{2}^{\beta_{2} / 2}}{(1-\rho) \sqrt{\Omega_{d 1} \Omega_{d 2}}}\right], \\
p_{r_{1} r_{2}}\left(r_{1}, r_{2}\right)= & \frac{\beta_{1} \beta_{2} r_{1}^{\beta_{1}-1} r_{2}^{\beta_{2}-1}}{\Omega_{c 1} \Omega_{c 2}(1-\rho)} \\
& \times \exp \left[-\frac{1}{1-\rho}\left(\frac{r_{1}^{\beta_{1}}}{\Omega_{c 1}}+\frac{r_{2}^{\beta_{2}}}{\Omega_{c 2}}\right)\right] \\
& \times I_{0}\left[\frac{2 \sqrt{\rho} r_{1}^{\beta_{1} / 2} r_{2}^{\beta_{2} / 2}}{(1-\rho) \sqrt{\Omega_{c 1} \Omega_{c 2}}}\right]
\end{aligned}
$$

while the joint multivariate PDFs of Weibull distributed random variables $R_{i}$ and $r_{i}, i=1, \ldots, L$, with exponential correlation can be expressed as [23, Equation (22)] as follows:

$$
\begin{aligned}
p_{R_{1} \cdots R_{L}} & \left(R_{1}, \ldots, R_{L}\right) \\
= & \frac{1}{(1-\rho)^{L-1}} \prod_{i=1}^{L} \frac{\beta_{i} R_{i}^{\beta_{i}-1}}{\Omega_{d i}} \\
& \times \exp \left\{-\frac{1}{1-\rho}\left[\frac{R_{1}^{\beta_{1}}}{\Omega_{d 1}}+\frac{R_{L}^{\beta_{L}}}{\Omega_{d L}}+(1+\rho) \sum_{i=2}^{L-1} \frac{R_{i}^{\beta_{i}}}{\Omega_{d i}}\right]\right\} \\
p_{r_{1} \cdots r_{L}}( & \left.r_{1}, \ldots, r_{L}\right) \\
= & \frac{1}{(1-\rho)}\left[\frac{2 \sqrt{\rho} I_{i}^{\beta_{i} / 2} R_{i+1}^{\beta_{i+1} / 2}}{(1-\rho) \sqrt[L]{\Omega_{d 1} \cdots \Omega_{d L}}}\right], \\
& \times \exp \left\{-\frac{1}{1-\rho}\left[\frac{r_{1}^{\beta_{1}}}{\Omega_{c 1}}+\frac{r_{L}^{\beta_{L}}}{\Omega_{c L}}+(1+\rho) \sum_{i=2}^{L-1} \frac{\beta_{i}^{\beta_{i}-1}}{\Omega_{c i}}\right]\right\} \\
& \times \prod_{i=1}^{L-1} I_{0}\left[\frac{2 \sqrt{\rho} r_{i}^{\beta_{i} / 2} r_{i+1}^{\beta_{i+1} / 2}}{(1-\rho) \sqrt[L]{\Omega_{c 1} \cdots \Omega_{c L}}}\right],
\end{aligned}
$$

where $\beta_{i}$ is the Weibull parameter. Weibull parameter expresses the fading severity $\left(\beta_{i}>0\right)$. As it increases, the severity of fading decreases, while for $\beta_{i}=2$, the Weibull distribution reduces to the Rayleigh distribution. The joint PDF of ratios of Weibull random variables, $\mu_{i}=R_{i} / r_{i}, i=$ $1, \ldots, L$, can be obtained as

$$
\begin{aligned}
& p_{\mu_{1} \cdots \mu_{L}}\left(\mu_{1}, \ldots, \mu_{L}\right) \\
& =\underbrace{\int_{0}^{\infty} \cdots \int_{0}^{\infty}|J| p_{R_{1} \cdots R_{L}}\left(\mu_{1} r_{1}, \ldots, \mu_{L} r_{L}\right)}_{L \text {-fold }} \\
& \quad \times p_{r_{1} \cdots r_{L}}\left(r_{1}, \ldots, r_{L}\right) d r_{1} \cdots d r_{L},
\end{aligned}
$$

where $|J|$ is the Jacobian transformation given by

$$
|J|=\left|\begin{array}{ccc}
\frac{d R_{1}}{d \mu_{1}} & \ldots & \frac{d R_{1}}{d \mu_{L}} \\
\vdots & & \\
\frac{d R_{L}}{d \mu_{1}} & & \frac{d R_{L}}{d \mu_{L}}
\end{array}\right|=\prod_{i=1}^{L} r_{i}
$$

Substituting the adequate joint PDFs of $R_{i}$ and $r_{i}$ in (17) and using the infinite-series representation of the modified Bessel function, after integrations, the joint bivariate PDF of 
$\mu_{1}$ and $\mu_{2}$ can be written in the following form [14, Equation (7)]:

$$
\begin{aligned}
& p_{\mu_{1} \mu_{2}}\left(\mu_{1}, \mu_{2}\right) \\
& =(1-\rho)^{2} \beta_{1} \beta_{2} \\
& \quad \times \sum_{k, \ell=0}^{\infty} \frac{\rho^{k+\ell} \mu_{1}^{(1+k) \beta_{1}-1} \mu_{2}^{(1+k) \beta_{2}-1} \Gamma^{2}(2+k+\ell)}{\left(\Omega_{d 1} \Omega_{d 2}\right)^{1+k}\left(\Omega_{c 1} \Omega_{c 2}\right)^{1+\ell}(k ! \ell !)^{2}} \\
& \quad \times\left(\left(\frac{\mu_{1}^{\beta_{1}}}{\Omega_{d 1}}+\frac{1}{\Omega_{c 1}}\right)\left(\frac{\mu_{2}^{\beta_{2}}}{\Omega_{d 2}}+\frac{1}{\Omega_{c 2}}\right)\right)^{-(2+k+\ell)}
\end{aligned}
$$

while the joint multivariate PDF of $\left\{\mu_{i}\right\}_{i=1}^{L}$ can be written as in $[16$, Equation (4)] as

$$
\begin{aligned}
& p_{\mu_{1} \cdots \mu_{L}}\left(\mu_{1}, \ldots, \mu_{L}\right) \\
& =(1-\rho)^{2} \\
& \times \prod_{i=1}^{L} \beta_{i} \sum_{k_{1}, \ldots, k_{L-1}, \ell_{1}, \ldots, \ell_{L-1}=0}^{\infty} \frac{\rho^{\sum_{i=1}^{L-1}\left(k_{i}+\ell_{i}\right)}}{(1+\rho)^{\sum_{i=2}^{L-1}\left(2+k_{i}+k_{i-1}+\ell_{i}+\ell_{i-1}\right)}} \\
& \times\left(\mu_{1}^{\left(1+k_{1}\right) \beta_{1}-1} \mu_{L}^{\left(1+k_{L-1}\right) \beta_{L}-1} \Gamma\left(2+k_{1}+\ell_{1}\right)\right. \\
& \times \Gamma\left(2+k_{L-1}+\ell_{L-1}\right) \\
& \times\left(\prod_{i=1}^{L}\left(\Omega_{d i}^{1+(2 / L) \sum_{j=1}^{L-1} k_{j}} \Omega_{c i}^{1+(2 / L) \sum_{j=1}^{L-1} \ell_{j}}\right)\right. \\
& \times\left(\frac{\mu_{1}^{\beta_{1}}}{\Omega_{d 1}}+\frac{1}{\Omega_{c 1}}\right)^{2+k_{1}+\ell_{1}} \\
& \left.\left.\times\left(\frac{\mu_{L}^{\beta_{L}}}{\Omega_{d L}}+\frac{1}{\Omega_{c L}}\right)^{2+k_{L-1}+\ell_{L-1}} \prod_{i=1}^{L-1}\left(k_{i} ! \ell_{i} !\right)^{2}\right)^{-1}\right) \\
& \times \prod_{i=2}^{L-1}\left[\mu_{i}^{\beta_{i}\left(1+k_{i}+k_{i-1}\right)-1}\left(\frac{\mu_{i}^{\beta_{i}}}{\Omega_{d i}}+\frac{1}{\Omega_{c i}}\right)^{-\left(2+k_{i}+k_{i-1}+\ell_{i}+\ell_{i-1}\right)}\right. \\
& \left.\times \Gamma\left(2+k_{i}+k_{i-1}+\ell_{i}+\ell_{i-1}\right)\right] \text {. }
\end{aligned}
$$

(b) Product Moments. Product moments are also important in wireless communications systems because they are a useful statistical tool to characterize a distribution. The $\left(\sum_{i=1}^{L} n_{i}\right)$ thorder moment of the product of $\left\{\mu_{i}\right\}_{i=1}^{L}$ can be derived as

$$
\begin{aligned}
& \varepsilon\left\langle\prod_{i=1}^{L} \mu_{i}^{n_{i}}\right\rangle \\
& =\underbrace{\int_{0}^{\infty} \cdots \int_{0}^{\infty}\left(\prod_{i=1}^{L} \mu_{i}^{n_{i}}\right) p_{\mu_{1} \cdots \mu_{L}}\left(\mu_{1}, \ldots, \mu_{L}\right) d \mu_{1} \cdots d \mu_{L} .}_{L \text {-fold }}
\end{aligned}
$$

Substituting the adequate joint PDF of ratios of Weibull distributed random variables in (21) and using [18, Equation (3.241/4)], for $n_{i} / \beta_{i}<1, i=1, \ldots, L$, product moments of two and multiple ratios of Weibull random variables are

$$
\begin{aligned}
\varepsilon\left\langle\mu_{1}^{n_{1}} \mu_{2}^{n_{2}}\right\rangle=U \sum_{k, \ell=0}^{\infty} & \frac{\rho^{k+\ell}}{(k ! \ell !)^{2}} \Gamma\left(1+k+\frac{n_{1}}{\beta_{1}}\right) \Gamma\left(1+k+\frac{n_{2}}{\beta_{2}}\right) \\
& \times \Gamma\left(1+\ell-\frac{n_{1}}{\beta_{1}}\right) \Gamma\left(1+\ell-\frac{n_{2}}{\beta_{2}}\right),
\end{aligned}
$$

where $U=(1-\rho)^{2}\left(\Omega_{d 1} / \Omega_{c 1}\right)^{n_{1} / \beta_{1}}\left(\Omega_{d 2} / \Omega_{c 2}\right)^{n_{2} / \beta_{2}}$ and

$$
\begin{aligned}
& \varepsilon\left\langle\prod_{i=1}^{L} \mu_{i}^{n_{i}}\right\rangle \\
& =(1-\rho)^{2} \\
& \times \sum_{k_{1}, \ldots, k_{L-1}, \ell_{1}, \ldots, \ell_{L-1}=0}^{\infty}\left(\rho^{\sum_{i=1}^{L-1}\left(k_{i}+\ell_{i}\right)} \Omega_{d 1}^{k_{1}-(2 / L) \sum_{i=1}^{L-1} k_{i}+\left(n_{1} / \beta_{1}\right)}\right. \\
& \times \Omega_{c 1}^{\ell_{1}-(2 / L) \sum_{i=1}^{L-1} \ell_{i}-\left(n_{1} / \beta_{1}\right)} \\
& \left.\times\left((1-\rho)^{\sum_{i=2}^{L-1}\left(2+k_{i}+k_{i-1}+\ell_{i}+\ell_{i-1}\right)}\right)^{-1}\right) \\
& \times \frac{\Omega_{d L}^{k_{L-1}-(2 / L) \sum_{i=1}^{L-1} k_{i}+\left(n_{L} / \beta_{L}\right)} \Omega_{c L}^{\ell_{L-1}-(2 / L) \sum_{i=1}^{L-1} \ell_{i}-\left(n_{L} / \beta_{L}\right)}}{\prod_{i=1}^{L-1}\left(k_{i} ! \ell_{i} !\right)^{2}} \\
& \times \Gamma\left(1+k_{1}+\frac{n_{1}}{\beta_{1}}\right) \Gamma\left(1+k_{L-1}+\frac{n_{L}}{\beta_{L}}\right) \\
& \times \Gamma\left(1+\ell_{1}-\frac{n_{1}}{\beta_{1}}\right) \Gamma\left(1+\ell_{L-1}-\frac{n_{L}}{\beta_{L}}\right)
\end{aligned}
$$




$$
\begin{aligned}
\times \prod_{i=2}^{L-1}\left\{\Omega_{d i}^{k_{i}+k_{i-1}-(2 / L) \sum_{j=1}^{L-1} k_{j}+\left(n_{i} / \beta_{i}\right)} \Omega_{c i}^{\ell_{i}+\ell_{i-1}-(2 / L) \sum_{j=1}^{L-1} \ell_{j}-\left(n_{i} / \beta_{i}\right)}\right. \\
\times \Gamma\left(1+k_{i}+k_{i-1}+\frac{n_{i}}{\beta_{i}}\right) \\
\left.\times \Gamma\left(1+\ell_{i}+\ell_{i-1}-\frac{n_{i}}{\beta_{i}}\right)\right\}
\end{aligned}
$$

respectively.

(c) PDF of Maximum. The PDF expression of maximum of ratios of random variables, $\mu_{\max }=\max \left\{\mu_{1}, \mu_{2}, \ldots, \mu_{L}\right\}$, can be derived as

$$
\begin{aligned}
p_{\mu_{\max }}(\mu) & \\
= & \underbrace{\iint_{0}^{\mu} \cdots \int_{0}^{\mu}}_{(L-1) \text {-fold }} p_{\mu_{1} \mu_{2} \cdots \mu_{L}}\left(\mu, \mu_{2}, \ldots, \mu_{L}\right) d \mu_{2} d \mu_{3} \cdots d \mu_{L} \\
& +\underbrace{\iint_{0}^{\mu} \cdots \int_{0}^{\mu}}_{(L-1) \text {-fold }} p_{\mu_{1} \mu_{2} \cdots \mu_{L}}\left(\mu_{1}, \mu, \ldots, \mu_{L}\right) d \mu_{1} d \mu_{3} \cdots d \mu_{L} \\
& \vdots \\
& +\underbrace{\iint_{0}^{\mu} \cdots \int_{0}^{\mu}}_{(L-1) \text {-fold }} p_{\mu_{1} \mu_{2} \cdots \mu_{L}}\left(\mu_{1}, \mu_{2}, \ldots, \mu\right) d \mu_{1} d \mu_{2} \cdots d \mu_{L-1} .
\end{aligned}
$$

Substituting the joint PDF of $\left\{\mu_{i}\right\}_{i=1}^{L}$ in the previous expression and after integrations, the PDFs of maximum of two and multiple ratios of Weibull random variables can be written, respectively, as in $[14, \operatorname{Equation}(11)]$ and $[16$, Equation(12)] as follows:

$$
\begin{aligned}
& p_{\mu_{\max }}(\mu) \\
&=(1-\rho)^{2} \sum_{k, \ell=0}^{\infty} \frac{\rho^{k+\ell} \Gamma^{2}(2+k+\ell) \mu^{(1+k)\left(\beta_{1}+\beta_{2}\right)-1}}{(1+k)(k ! \ell !)^{2}}\left(\frac{\Omega_{c 1} \Omega_{c 2}}{\Omega_{d 1} \Omega_{d 2}}\right)^{1+k} \\
& \times\left\{\frac{\beta_{1}}{\left(1+\left(\left(\Omega_{c 1} / \Omega_{d 1}\right) \mu^{\beta_{1}}\right)\right)^{2+k+\ell}}\right. \\
& \quad \times{ }_{2} F_{1}\left(2+k+\ell, 1+k, 2+k ;-\frac{\Omega_{c 2}}{\Omega_{d 2}} \mu^{\beta_{2}}\right) \\
& \quad+\frac{\beta_{2}}{\left(1+\left(\left(\Omega_{c 2} / \Omega_{d 2}\right) \mu^{\beta_{2}}\right)\right)^{2+k+\ell}} \\
&\left.\quad \times{ }_{2} F_{1}\left(2+k+\ell, 1+k, 2+k ;-\frac{\Omega_{c 1}}{\Omega_{d 1}} \mu^{\beta_{1}}\right)\right\}
\end{aligned}
$$

$$
\begin{aligned}
& p_{\mu_{\max }}(\mu) \\
& =(1-\rho)^{2} \\
& \quad \times \sum_{k_{1}, k_{2}, \ldots, k_{L-1}, \ell_{1}, \ell_{2}, \ldots, \ell_{L-1}=0}^{\infty} \frac{\Omega_{c 1}^{1+k_{1}+\ell_{1}-(2 / L) \sum_{i=1}^{L-1} \ell_{i}} \Omega_{c L}^{1+k_{L-1}+\ell_{L-1}-(2 / L) \sum_{i=1}^{L-1} \ell_{i}}}{(1+\rho)^{\sum_{i=2}^{L-1}\left(2+k_{i}+k_{i-1}+\ell_{i}+\ell_{i-1}\right)}} \\
& \quad \times \frac{\Gamma\left(2+k_{1}+\ell_{1}\right) \Gamma\left(2+k_{L-1}+\ell_{L-1}\right)}{\left(\prod_{i=1}^{L} \Omega_{d i}\right)^{1+(2 / L) \sum_{i=1}^{L-1} k_{i}} \prod_{i=0}^{L-1}\left(k_{i} ! \ell_{i} !\right)^{2}} \\
& \quad \times \rho^{\sum_{i=1}^{L-1}\left(k_{i}+\ell_{i}\right)} \mu^{\left(1+k_{1}\right) \beta_{1}+\left(1+k_{L-1}\right) \beta_{L}+\sum_{i=2}^{L-1}\left(1+k_{i}+k_{i-1}\right) \beta_{i}-1} \\
& \quad \times \prod_{i=2}^{L-1}\left\{\Omega_{c i}^{1+k_{i}+k_{i-1}+\ell_{i}+\ell_{i-1}-(2 / L) \sum_{i=1}^{L-1} \ell_{i}}\right. \\
& \left.\quad \times \Gamma\left(2+k_{i}+k_{i-1}+\ell_{i}+\ell_{i-1}\right)\right\} Z(\mu),
\end{aligned}
$$

respectively, where

$$
\begin{aligned}
& Z(\mu) \\
& =\left(\frac{\beta_{1}}{\left(1+k_{L-1}\right)\left(1+\left(\left(\Omega_{c 1} / \Omega_{d 1}\right) \mu^{\beta_{1}}\right)\right)^{2+k_{1}+\ell_{1}}}\right. \\
& \times{ }_{2} F_{1}\left(2+k_{L-1}+\ell_{L-1}, 1+k_{L-1} ; 2+k_{L-1} ;-\frac{\Omega_{c L}}{\Omega_{d L}} \mu^{\beta_{L}}\right) \\
& +\frac{\beta_{L}}{\left(1+k_{1}\right)\left(1+\left(\left(\Omega_{c L} / \Omega_{d L}\right) \mu^{\beta_{L}}\right)\right)^{2+k_{L-1}+\ell_{L-1}}} \\
& \left.\times{ }_{2} F_{1}\left(2+k_{1}+\ell_{1}, 1+k_{1} ; 2+k_{1} ;-\frac{\Omega_{c 1}}{\Omega_{d 1}} \mu^{\beta_{1}}\right)\right) \\
& \times \prod_{i=2}^{L-1}\left({ } _ { 2 } F _ { 1 } \left(2+k_{i}+k_{i-1}+\ell_{i}+\ell_{i-1}, 1+k_{i}+k_{i-1}\right.\right. \text {; } \\
& \left.2+k_{i}+k_{i-1} ;-\frac{\Omega_{c i}}{\Omega_{d i}} \mu^{\beta_{i}}\right) \\
& \left.\times\left(1+k_{i}+k_{i-1}\right)^{-1}\right) \\
& +\left({ }_{2} F_{1}\left(2+k_{1}+\ell_{1}, 1+k_{1} ; 2+k_{1} ;-\frac{\Omega_{c 1}}{\Omega_{d 1}} \mu^{\beta_{1}}\right)\right. \\
& \times{ }_{2} F_{1}\left(2+k_{L-1}+\ell_{L-1}, 1+k_{L-1} ; 2+k_{L-1} ;\right. \\
& \left.-\frac{\Omega_{c L}}{\Omega_{d L}} \mu^{\beta_{L}}\right) \\
& \left.\times\left(\left(1+k_{1}\right)\left(1+k_{L-1}\right)\right)^{-1}\right) \\
& \times \sum_{j=2}^{L-1} \frac{\beta_{j}}{\left(1+\left(\left(\Omega_{c j} / \Omega_{d j}\right) \mu^{\beta_{j}}\right)\right)^{2+k_{j}+k_{j-1}+\ell_{j}+\ell_{j-1}}} \\
& \times \prod_{i=2 ; i \neq j}^{L-1}\left[{ } _ { 2 } F _ { 1 } \left(2+k_{i}+k_{i-1}+\ell_{i}+\ell_{i-1}, 1+k_{i}+k_{i-1}\right.\right. \\
& \left.2+k_{i}+k_{i-1} ;-\frac{\Omega_{c i}}{\Omega_{d i}} \mu^{\beta_{i}}\right) \\
& \left.\times\left(1+k_{i}+k_{i-1}\right)^{-1}\right] \text {. }
\end{aligned}
$$




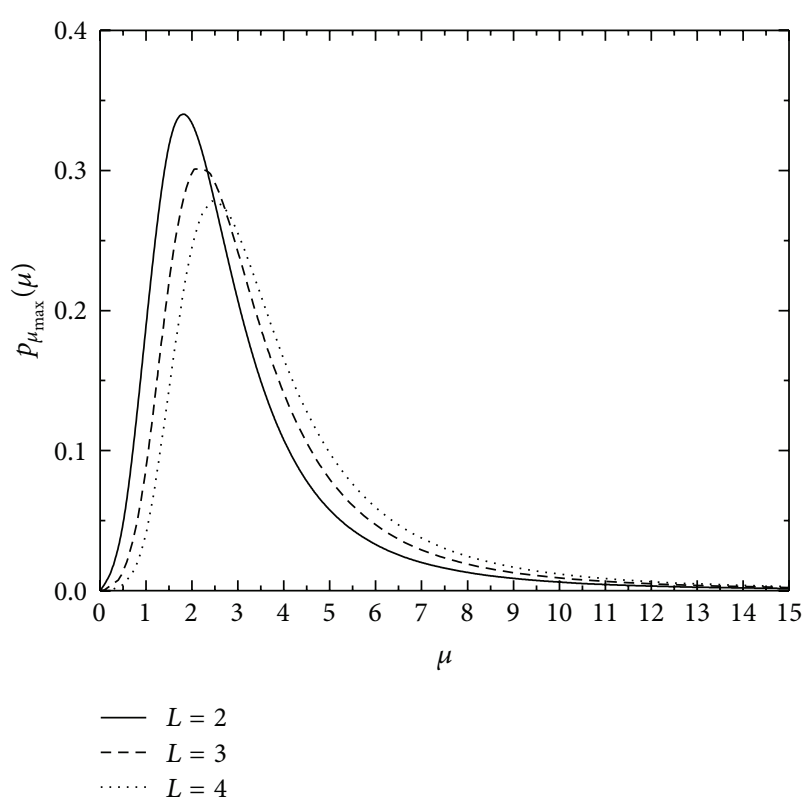

FIGURE 1: Probability density function of maximum of ratios of Weibull random variables for $\rho=0.2, \Omega_{d i}=0.8, \Omega_{c i}=0.2$, and $\beta_{i}=2.5, i=1, \ldots, L$.

Figure 1 illustrates the PDF of maximum of two, three, and four ratios of random variables.

(d) PDF of Minimum. The minimum of ratios of Weibull random variables, $\mu_{\text {min }}=\min \left\{\mu_{1}, \mu_{2}, \ldots, \mu_{L}\right\}$, can be obtained by

$$
\begin{aligned}
& p_{\mu_{\min }}(\mu) \\
& =\underbrace{\iint_{\mu}^{\infty} \cdots \int_{\mu}^{\infty}}_{(L-1) \text {-fold }} p_{\mu_{1} \mu_{2} \cdots \mu_{L}}\left(\mu, \mu_{2}, \ldots, \mu_{L}\right) d \mu_{2} d \mu_{3} \cdots d \mu_{L} \\
& +\underbrace{\iint_{\mu}^{\infty} \cdots \int_{\mu}^{\infty} p_{\mu_{1} \mu_{2} \cdots \mu_{L}}\left(\mu_{1}, \mu, \ldots, \mu_{L}\right) d \mu_{1} d \mu_{3} \cdots \mu_{L}}_{(L-1) \text {-fold }} \\
& \quad \vdots \underbrace{\iint_{\mu}^{\infty} \cdots \int_{\mu}^{\infty}}_{(L-1) \text {-fold }} p_{\mu_{1} \mu_{2} \cdots \mu_{L}}\left(\mu_{1}, \mu_{2}, \ldots, \mu\right) d \mu_{1} d \mu_{2} \cdots d \mu_{L-1} .
\end{aligned}
$$

Substituting the adequate joint PDF in (28) and using [18, Equation $(3.194 / 2)]$, with $\left(\mu^{\beta_{i}} / \Omega_{d i}\right)+\left(1 / \Omega_{c i}\right)=\Phi_{i}$, $\Omega_{d i} / \Omega_{c i} \mu^{\beta_{i}}=\varphi_{i}, i=1,2, \ldots, L$, the PDF of minimum of two ratios of random variables becomes

$$
\begin{aligned}
p_{\mu_{\text {min }}}(\mu)= & (1-\rho)^{2} \\
& \times \sum_{k, \ell=0}^{\infty} \frac{\rho^{k+\ell} \Gamma^{2}(2+k+\ell)}{\left(\Omega_{d 1} \Omega_{d 2}\right)^{1+k}\left(\Omega_{c 1} \Omega_{c 2}\right)^{1+\ell}(k ! \ell !)^{2}(1+\ell)}
\end{aligned}
$$

$$
\begin{aligned}
& \times\left\{\beta_{1} \Omega_{d 2}^{2+k+\ell} \frac{\mu^{(1+k) \beta_{1}-(1+\ell) \beta_{2}-1}}{\Phi_{1}^{2+k+\ell}}\right. \\
& \quad \times{ }_{2} F_{1}\left(2+k+\ell, 1+\ell ; 2+\ell ;-\varphi_{2}\right) \\
& \quad+\beta_{2} \Omega_{d 1}^{2+k+\ell} \frac{\mu^{(1+k) \beta_{2}-(1+\ell) \beta_{1}-1}}{\Phi_{2}^{2+k+\ell}} \\
& \left.\quad \times{ }_{2} F_{1}\left(2+k+\ell, 1+\ell ; 2+\ell ;-\varphi_{1}\right)\right\},
\end{aligned}
$$

and finally, the PDF of minimum of multiple ratios of random variables can be expressed in a more complicated form as follows:

$$
\begin{aligned}
& p_{\mu_{\min }}(\mu) \\
& =(1-\rho)^{2} \\
& \times \sum_{k_{1}, k_{2}, \ldots, k_{L-1}, \ell_{1}, \ell_{2}, \ldots . \ell_{L-1}=0}^{\infty}\left(\rho^{\sum_{i=1}^{L-1}\left(k_{i}+\ell_{i}\right)}(1+\rho)^{-\sum_{i=2}^{L-1}\left(2+k_{i}+k_{i-1}+\ell_{i}+\ell_{i-1}\right)}\right. \\
& \times\left(\left(\prod_{i=1}^{L} \Omega_{d i}\right)^{1+(2 / L) \sum_{i=1}^{L-1} k_{i}}\right. \\
& \times\left(\prod_{i=2}^{L-1}\left(\frac{\Omega_{d i}^{2+k_{i}+k_{i-1}+\ell_{i}+\ell_{i-1}}}{1+\ell_{i}+\ell_{i-1}}{ }_{2} F_{1} \Omega_{c i}\right)^{1+(2 / L) \sum_{i=1}^{L-1} \ell_{i}}\right) \\
& \times\left(2+k_{1}+\ell_{1}\right) \Gamma\left(2+k_{L-1}+\ell_{L-1}\right) \\
& \times \prod_{i=2}^{L-1} \Gamma\left(2+k_{i}+k_{i-1}+\ell_{i}+\ell_{i-1}\right) \\
& \times\left(\prod_{i=1}^{L-1}\left(k_{i} ! \ell_{i} !\right)^{2}\right)^{-1} \\
& \left.\times\left(1+\ell_{i}+\ell_{i-1} ; 2+\ell_{i}+\ell_{i-1} ;-\varphi_{i}\right)\right)
\end{aligned}
$$$$
\begin{aligned}
\times\left(\frac{\beta_{1} \Omega_{d L}^{2+k_{L-1}+\ell_{L-1}} \mu^{\left(1+k_{1}\right) \beta_{1}-\sum_{i=2}^{L-1}\left(1+\ell_{i}+\ell_{i-1}\right) \beta_{i}-\left(1+\ell_{L-1}\right) \beta_{L}-1}}{\left(1+\ell_{L-1}\right) \Phi_{1}^{2+k_{1}+\ell_{1}}}\right. \\
\quad \times{ }_{2} F_{1}\left(2+k_{L-1}+\ell_{L-1}, 1+\ell_{L-1} ; 2+\ell_{L-1} ;-\varphi_{L}\right)
\end{aligned}
$$ 


$$
\begin{gathered}
+\frac{\beta_{L} \Omega_{d 1}^{2+k_{1}+\ell_{1}} \mu^{\left(1+k_{L-1}\right) \beta_{L}-\sum_{i=2}^{L-1}\left(1+\ell_{i}+\ell_{i-1}\right) \beta_{i}-\left(1+\ell_{1}\right) \beta_{1}-1}}{\left(1+\ell_{1}\right) \Phi_{L}^{2+k_{L-1}+\ell_{L-1}}} \\
\left.\times{ }_{2} F_{1}\left(2+k_{1}+\ell_{1}, 1+\ell_{1} ; 2+\ell_{1} ;-\varphi_{1}\right)\right) \\
+{ }_{2} F_{1}\left(2+k_{1}+\ell_{1}, 1+\ell_{1} ; 2+\ell_{1} ;-\varphi_{1}\right) \\
\times{ }_{2} F_{1}\left(2+k_{L-1}+\ell_{L-1} ; 1+\ell_{L-1} ; 2+\ell_{L-1} ;-\varphi_{L}\right) \\
\times \frac{\Omega_{d 1}^{2+k_{1}+\ell_{1}} \Omega_{d L}^{2+k_{L-1}+\ell_{L-1}}}{\left(1+\ell_{1}\right)\left(1+\ell_{L-1}\right)} \\
\times \sum_{i=2}^{L-1}\left[\frac{\beta_{i}}{\Phi_{i}^{2+k_{i}+k_{i-1}+\ell_{i}+\ell_{i-1}}}\right. \\
\times \prod_{j=2, j \neq i}^{L-1}\left(\frac{\Omega_{d j}^{2+k_{j}+k_{j-1}+\ell_{j}+\ell_{j-1}}}{1+\ell_{j}+\ell_{j-1}}\right. \\
\left.\left.\times \mu^{\left(1+k_{i}+k_{i-1}\right) \beta_{i}-\left(1+\ell_{1}\right) \beta_{1}-\left(1+\ell_{L-1}\right) \beta_{L}-\sum_{j=2, j \neq i}^{L-1}\left(1+\ell_{j}+\ell_{j-1}\right) \beta_{j}-1}\right]\right\} . \\
\times{ }_{2} F_{1}\left(2+k_{j}+k_{j-1}+\ell_{j}+\ell_{j-1},\right. \\
\left.\left.1+\ell_{j}+\ell_{j-1} ; 2+\ell_{j}+\ell_{j-1} ;-\varphi_{j}\right)\right)
\end{gathered}
$$

The PDF of minimum of ratios of random variables is important for analyzing multihop relayed communication systems in which the source terminal communicates with the destination terminal through a number of relay terminals [24]. In that case, $\mu_{i}$ is signal-to-interference ratio, $\Omega_{d i}$ and $\Omega_{c i}$ are the average signal desired and interference powers at $i$ th terminal input, respectively, and $\rho$ is the correlation between two successive terminals (in the case of independent terminals, which is the real scenario in practice, correlation coefficient tends to be zero). Figure 2 illustrates the PDF of minimum of two, three, and four ratios of random variables.

The main problem in the infinite-series expressions can be their convergence. Expressions presented in the paper converge rapidly, and thus they can be efficiently used. As an illustrative example, the number of terms needed to be summed in expressions for PDF of minimum of ratios of Weibull random variables is shown in Table 1. It is evident that the number of terms depends strongly on the correlation coefficient.

\section{Conclusion}

The distributions of ratios of random variables, $\mu_{i}=R_{i} / r_{i}$, $i=1, \ldots, L$, are of interest in many areas of science. In this paper, expressions for the joint PDF and PDF of maximum of two ratios of Rayleigh, Rician, and Nakagami- $m$ distributed random variables have been presented. Motivated by the

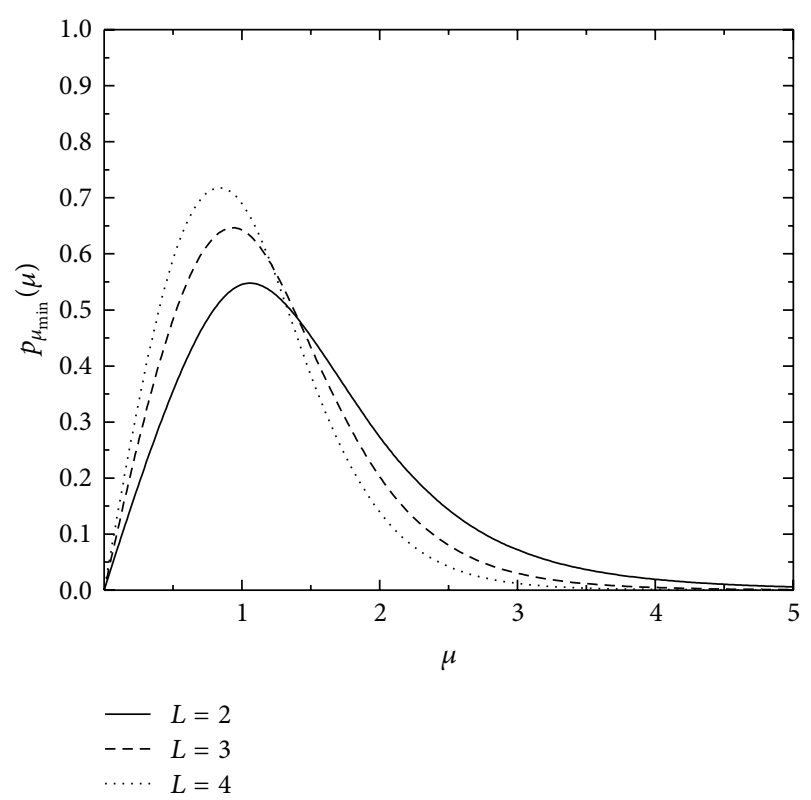

FIgURe 2: Probability density function of minimum of ratios of Weibull random variables for $\rho=0.2, \Omega_{d i}=0.8, \Omega_{c i}=0.2$, and $\beta_{i}=2.5, i=1, \ldots, L$.

TABLE 1: Number of terms needed to be summed to achieve accuracy at the 4th digit of expressions for PDF of minimum of ratios of Weibull random variables for $\Omega_{d i}=0.8, \Omega_{c i}=0.2, \beta_{i}=2.5$, $i=1, \ldots, L$.

\begin{tabular}{lccc}
\hline & $\mu=0.5$ & $\mu=1$ & $\mu=2$ \\
\hline$L=2, \rho=0.2$ & 7 & 5 & 5 \\
$L=2, \rho=0.6$ & 17 & 15 & 13 \\
$L=3, \rho=0.2$ & 8 & 6 & 5 \\
$L=3, \rho=0.6$ & 18 & 16 & 16 \\
\hline
\end{tabular}

fact that Weibull distribution exhibits an excellent fit to experimental fading channel measurements, special attention is dedicated to the case of ratios of Weibull random variables. For this case, expressions for the joint PDF, product moments, PDF of maximum, and PDF of minimum of arbitrary number of ratios of Weibull distributed random variables have been obtained. An application of these results for the wireless communications community has also been described.

\section{Acknowledgments}

The authors were supported in part by the Serbian Ministry of Education, Science and Technological Development. The authors are also indebted to the referees for the careful reading of the paper and their suggestions which have improved the paper.

\section{References}

[1] M. K. Simon and M. S. Alouini, Digital Communication over Fading Channels, John Wiley \& Sons, New York, NY, USA, 1st edition, 2000. 
[2] A. Goldsmith, Wireless Communications, Cambridge University Press, New York, NY, USA, 2005.

[3] V. A. Aalo, "Performance of maximal-ratio diversity systems in a correlated Nakagami-fading environment," IEEE Transactions on Communications, vol. 43, no. 8, pp. 2360-2369, 1995.

[4] V. V. Veeravalli, "On performance analysis for signaling on correlated fading channels," IEEE Transactions on Communications, vol. 49, no. 11, pp. 1879-1883, 2001.

[5] G. K. Karagiannidis, D. A. Zogas, and S. A. Kotsopoulos, "On the multivariate Nakagami- $m$ distribution with exponential correlation," IEEE Transactions on Communications, vol. 51, no. 8, pp. 1240-1244, 2003.

[6] S. Nadarajah and S. Kotz, "On the ratio of Pearson type VII and Bessel random variables," Journal of Applied Mathematics and Decision Sciences, no. 4, pp. 191-199, 2005.

[7] A. P. Basu and R. H. Lochner, "On the distribution of the ratio of two random variables having generalized life distributions," Technometrics, vol. 13, pp. 281-287, 1971.

[8] P. J. Korhonen and S. C. Narula, "The probability distribution of the ratio of the absolute values of two normal variables," Journal of Statistical Computation and Simulation, vol. 33, no. 3, pp. 173182, 1989.

[9] G. Marsaglia, "Ratios of normal variables and ratios of sums of uniform variables," Journal of the American Statistical Association, vol. 60, pp. 193-204, 1965.

[10] T. Pham-Gia, "Distributions of the ratios of independent beta variables and applications," Communications in Statistics, vol. 29, no. 12, pp. 2693-2715, 2000.

[11] D. V. Hinkley, "On the ratio of two correlated normal random variables," Biometrika, vol. 56, pp. 635-639, 1969.

[12] R. Y. Lee, B. S. Holland, and J. A. Flueck, "Distribution of a ratio of correlated gamma random variables," SIAM Journal on Applied Mathematics, vol. 36, no. 2, pp. 304-320, 1979.

[13] G. K. Karagiannidis, "Performance analysis of SIR-based dual selection diversity over correlated Nakagami- $m$ fading channels," IEEE Transactions on Vehicular Technology, vol. 52, no. 5, pp. 1207-1216, 2003.

[14] M. Č. Stefanović, D. M. Milović, A. M. Mitić, and M. M. Jakovljević, "Performance analysis of system with selection combining over correlated Weibull fading channels in the presence of cochannel interference," AEU-International Journal of Electronics and Communications, vol. 62, pp. 695-700, 2008.

[15] A. S. Panajotović, M. Č. Stefanović, and D. L. Drača, "Performance analysis of system with selection combining over correlated Rician fading channels in the presence of cochannel interference," AEU-International Journal of Electronics and Communications, vol. 63, no. 12, pp. 1061-1066, 2009.

[16] M. Č. Stefanović, D. L. Drača, A. S. Panajotović, and N. M. Sekulović, "Performance analysis of system with L-branch selection combining over correlated Weibull fading channels in the presence of cochannel interference," International Journal of Communication Systems, vol. 23, no. 2, pp. 139-150, 2010.

[17] A. V. Mosić, M. Č. Stefanović, S. R. Panić, and A. S. Panajotović, "Performance analysis of dual-branch selection combining over correlated rician fading channels for desired signal and cochannel interference," Wireless Personal Communications, vol. 55, pp. 475-484, 2009.

[18] I. S. Gradshteyn and I. M. Ryzhik, Table of Integrals, Series, and Products, Academic Press, San Diego, Calif, USA, 5th edition, 1996, CD version 1.0.
[19] M. Nakagami, "The $m$-distribution-a general formula of intensity distribution of rapid fading," in Statistical Methods in Radio Wave Propagation, pp. 3-36, Pergamon, Oxford, UK, 1960.

[20] F. Babich and G. Lombardi, "Statistical analysis and characterization of the indoor propagation channel," IEEE Transactions on Communications, vol. 48, no. 3, pp. 455-464, 2000.

[21] G. Tzeremes and C. G. Christodoulou, "Use of Weibull distribution for describing outdoor multipath fading," in Proceedings of the IEEE Antennas and Propagation Society International Symposium, vol. 1, pp. 232-235, San Antonio, Tex, USA, June 2002.

[22] N. H. Shepherd, "Radio wave loss deviation and shadow loss at $900 \mathrm{MHz}$," IEEE Transactions on Vehicular Technology, vol. 26, no. 4, pp. 309-313, 1977.

[23] N. C. Sagias and G. K. Karagiannidis, "Gaussian class multivariate Weibull distributions: theory and applications in fading channels," IEEE Transactions on Information Theory, vol. 51, no. 10, pp. 3608-3619, 2005.

[24] E. Mekić, M. Stefanović, P. Spalević, N. Sekulović, and A. Stanković, "Statistical analysis of ratio of random variables and its application in performance analysis of multihop wireless transmissions," Mathematical Problems in Engineering, vol. 2012, Article ID 841092, 10 pages, 2012. 


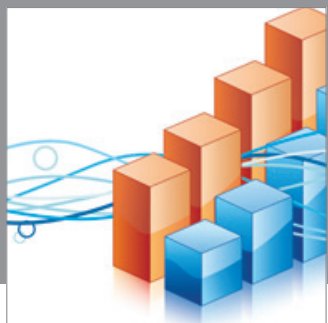

Advances in

Operations Research

mansans

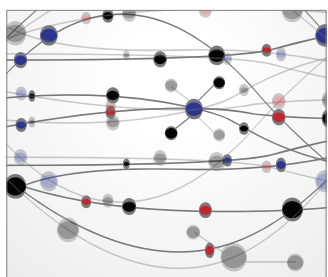

The Scientific World Journal
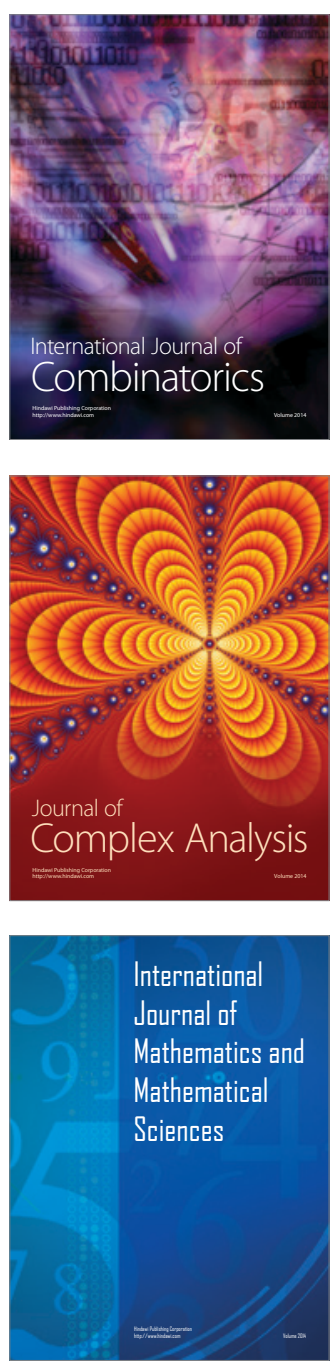
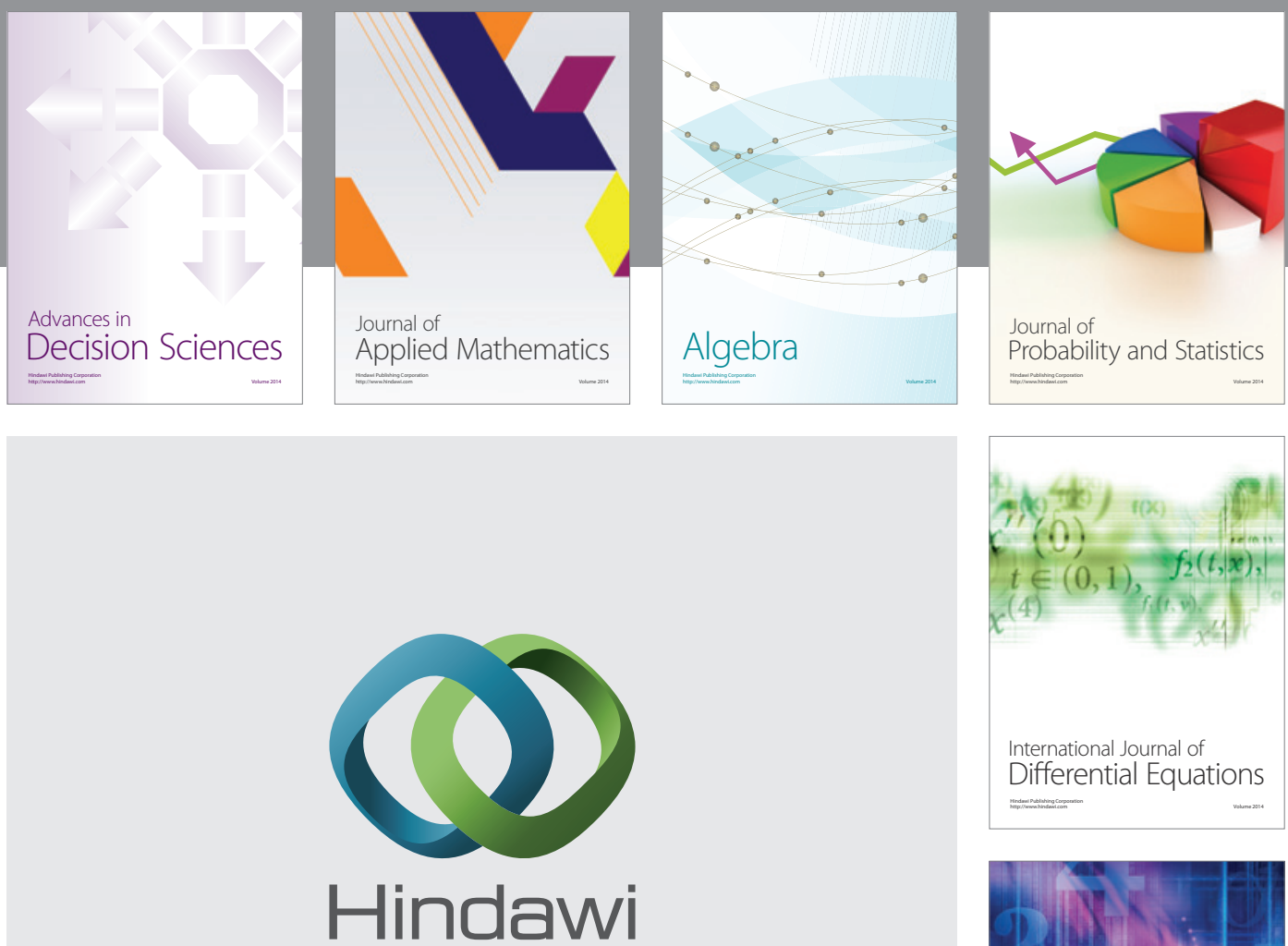

Submit your manuscripts at http://www.hindawi.com
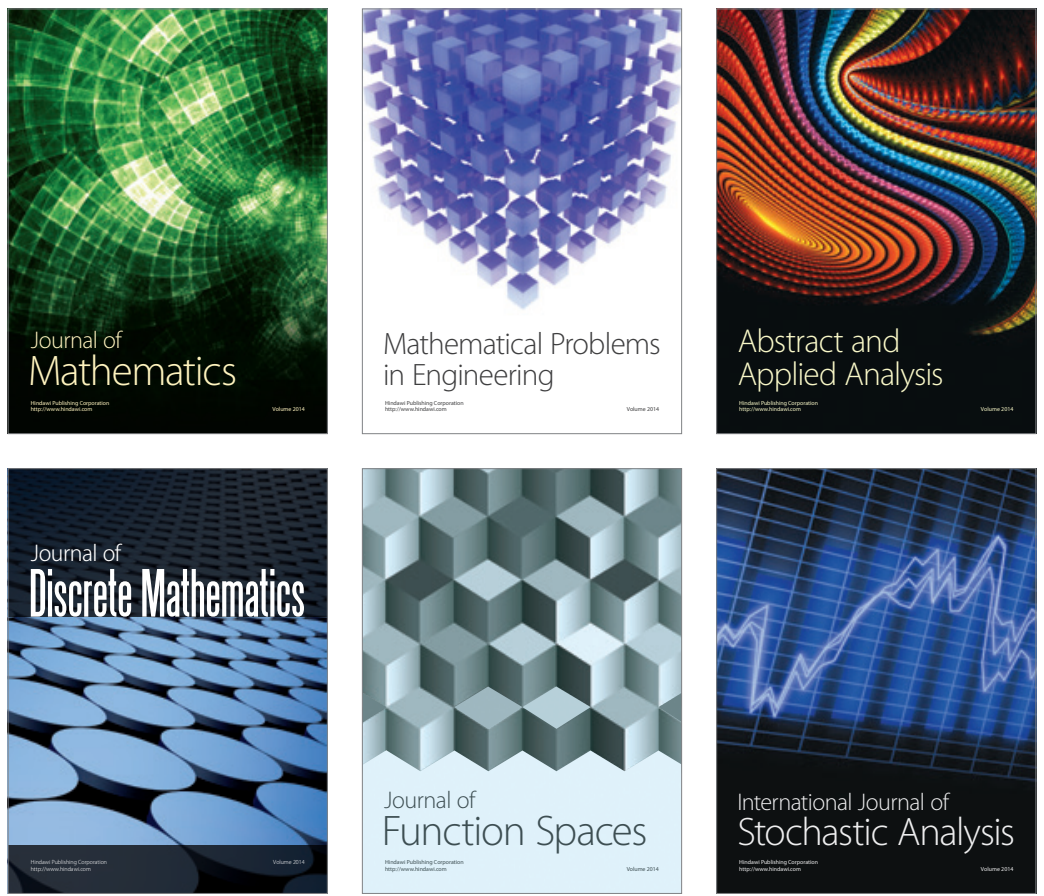

Journal of

Function Spaces

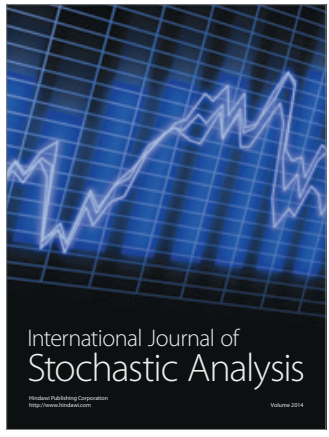

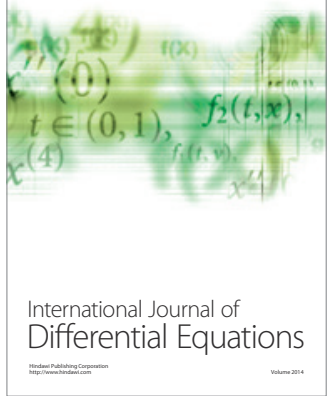
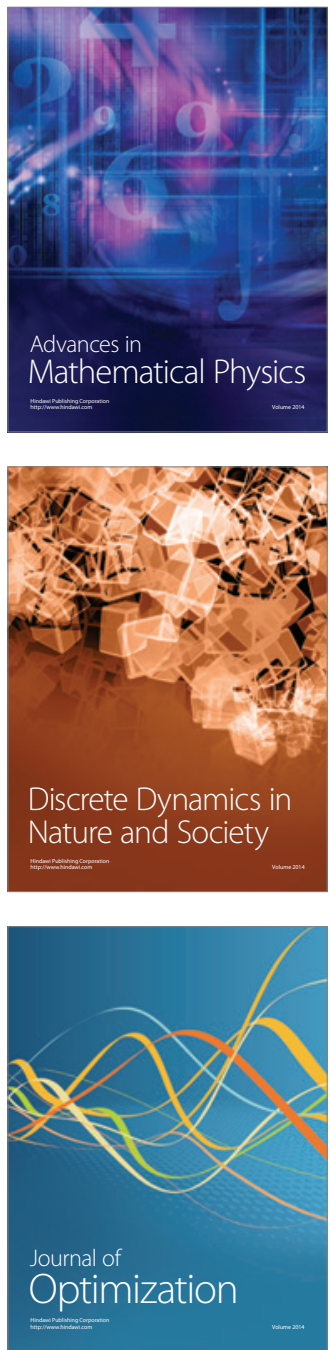